\title{
Crystal and molecular structures of small-molecule solids solved with the free-distribution software PSSP and WinPSSP
}

\author{
S. Pagola ${ }^{1}$, A. Polymeros ${ }^{2}$, N. Kourkoumelis ${ }^{3}$ \\ ${ }^{I}$ Department of Chemistry \& Biochemistry, Old Dominion University, Norfolk, VA, 23529, USA, \\ ${ }^{2}$ University of Ioannina, Department of Physics, Ioannina, 45110, Greece, \\ ${ }^{3}$ University of Ioannina, Department of Medical Physics, Ioannina, 45110, Greece \\ spagola@odu.edu
}

Since the early 2000's several free-distribution and commercial computer programs have advanced crystal structure determination from powders (SDPD). The free-distribution software WinPSSP [1] consists of a graphical user interface for an essentially unmodified version of PSSP [2], which uses a reconstructed X-ray powder diffraction pattern (a pseudo-pattern) made of correlated peak intensities calculated from the results of a Le Bail fit [3] (FWHM, peak positions, multiplicity factors and squared structure-factor amplitudes). The simulated annealing algorithm [4] is used to locate the asymmetric unit (provided in Cartesian coordinates) in the unit cell, using a space group symmetry candidate, a cost function (quantitatively evaluating the agreement between the diffracted intensity in the pseudo-pattern and that calculated from a large number of trial models), once a set of structural parameters (fragment positions, Eulerian angles and torsional angles) have been defined by the user. The crystal and molecular structures of more than fifty small-molecule organics have been solved with PSSP and WinPSSP. After Rietveld refinement (typically done with GSAS [5]), those have been published in peer-reviewed journals, or the atomic coordinates have been submitted together with reference powder diffraction patterns to the Powder Diffraction File database [6]. In most cases, synchrotron X-ray powder diffraction from capillary transmission geometry has been used.

This work analyses the distribution of various indicators among the structures solved, such as internal, external and total degrees of freedom, space group symmetries, Z, Z', unit cell volumes, number of atoms in the asymmetric unit (with and without hydrogens) and number of fragments to independently locate. The uses of solid-state characterization techniques providing information that enabled or confirmed SDPD (thermogravimetry, differential scanning calorimetry, NMR, optical microscopy and DFT calculations) is also highlighted. SDPD common impediments (e.g., preferred orientation from laboratory powder diffraction data) will be indicated.

Even though simple structures can be solved in the order of minutes using WinPSSP [2], due to the approximately exponential increase of the number of trial models required while the number of structural parameters increases [2], a challenge for the current search algorithm is to simultaneously and correctly locate many crystallographically independent fragments (typically three or more) using a reasonable number of trial models, comparable with that used in SDPD with small to moderate size asymmetric units. WinPSSP [2] facilitates the relocation of fragments incorrectly positioned in initial runs (as deemed by the user, considering data from varied solid-state characterization techniques and likely chemical bonding), and examples of this capability toward solving large structures will be discussed.

Another work goal is to attract undergraduate students to SDPD, whenever possible reducing the black box use of crystallography software. Guided SDPD examples have been presented in two workshops and will be used in a yearly SDPD workshop at Old Dominion University.

[1] Pagola, S., Polymeros, A. \& Kourkoumelis, N. (2017). J. Appl. Cryst. 50, 293-303.

[2] Pagola, S. \& Stephens, P. W. (2010). J. Appl. Cryst. 43, 370-376.

[3] Le Bail, A. (2005). Powder Diffraction 20, 316-326.

[4] Kirkpatrick, S., Gellat, C. D. \& Vecchi, M. P. (1983). Science 220, 671-680.

[5] Larson, A. C. \& Von Dreele, R. B. (2004). General Structure Analysis System (GSAS). Los Alamos National Laboratory Report 86-748.

[6] Gates-Rector, S. \& Blanton, T. (2019). Powder Diffraction 34, 352-360.

\section{Keywords: powder diffraction; direct-space methods; simulated annealing; SDPD; crystal structure solution from powders}

Use of the National Synchrotron Light Source, Brookhaven National Laboratory, was supported by the U.S. Department of Energy, Office of Science, Office of Basic Energy Sciences, under Contract No. DE-AC02-98CH10886. This research used resources of the Advanced Photon Source, a U.S. Department of Energy (DOE) Office of Science User Facility operated for the DOE Office of Science by Argonne National Laboratory under Contract No. DE-AC02-06CH11357. SP gratefully acknowledges partial funding from the International Centre for Diffraction Data (ICDD) through GIA 08-04 and workshop sponsorship, and partial funding and other support from the Chemistry \& Biochemistry Department at Old Dominion University. 